Pontifícia Universidade Católica $_{\text {na }}$

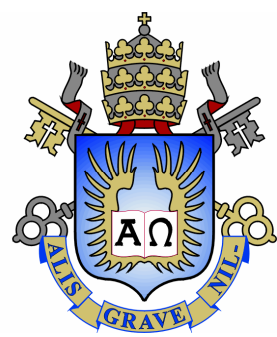

Roseli Martins de Souza

\title{
DESENVOLVIMENTO DE MÉTODOS ANALÍTICOS PARA DETERMINAÇÃO DE ELEMENTOS-TRAÇO EM AMOSTRAS OLEOSAS E PASTOSAS POR ICP OES E ICP- MS
}

Tese de Doutorado

Tese apresentada ao Programa de Pós-graduação em Química do Departamento de Química da PUC-Rio como parte dos requisitos parciais para obtenção do título de Doutor em Química.

Orientador: Ricardo Queiroz Aucélio Co-Orientador: Carmem Lúcia Porto da Silveira

Rio de Janeiro

Março de 2007 


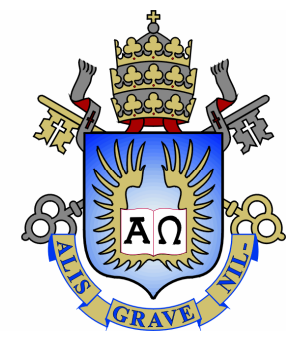

Roseli Martins de Souza

\section{Desenvolvimento de métodos analíticos para determinação de elementos-traço em amostras oleosas e pastosas por ICP OES e ICP- MS}

\begin{abstract}
Tese apresentada como requisito parcial para obtenção do título de Doutora em Química Analítica pelo Programa de Pós-Graduação em Química da PUC - Rio. Aprovada pela Comissão Examinadora abaixo
\end{abstract} assinada.

Prof. Ricardo Queiroz Aucélio Orientador Departamento de Química - PUC - Rio

Profa. Carmem L. Porto da Silveira Co-Orientadora Departamento de Química - PUC - Rio

Profa. Isabel Maria N. da Silva Moreira Departamento de Química - PUC - Rio

Prof. Norbert F. Miekeley Departamento de Química - PUC - Rio

Prof. Adilson José Curtius Centro de Ciências Físicas e Matemáticas Departamento de Química - UFSC

Prof. Ricardo Erthal Santelli Departamento de Geoquímica - UFF

Dr. Valnei Smarçaro da Cunha INMETRO - Rio

Prof. José Eugenio Leal Coordenador Setorial de Pós-Graduação do Centro Técnico Científico - PUC - Rio Rio de Janeiro, 26 de março de 2007 
Todos os direitos reservados. É proibida a reprodução total ou parcial do trabalho sem autorização da universidade, da autora e dos orientadores.

Roseli Martins de Souza

Graduou-se em Engenharia Química na Universidade Federal Rural do Rio de Janeiro (UFRRJ) em 1995. Obteve o título de Tecnólogo em Cervejaria pelo Senai em 1996. Desenvolveu projeto na área de espessantes associativos para perfuração de poços de petróleo e, posteriormente, supervisão, controle de qualidade e implantação do programa de qualidade total em processos da indústria cervejeira e alimentícia. Obteve o título de Mestre em Química pela PUC-Rio em 2003. Desenvolveu projeto na área de desenvolvimento de métodos analíticos para determinação de elementos refratários em amostras de petróleo.

Ficha Catalográfica

Souza, Roseli Martins de

Desenvolvimento de métodos analíticos para determinação de elementos-traço em amostras oleosas e pastosas por ICP OES e ICP-MS / Roseli Martins de Souza ; orientador: Ricardo Queiroz Aucélio; coorientador: Carmen Lúcia Porto da Silveira. - 2007.

187 f. : il. ; $30 \mathrm{~cm}$

Tese (Doutorado em Química)-Pontifícia Universidade Católica do Rio de Janeiro, Rio de Janeiro, 2007.

Inclui bibliografia

1. Química - Teses. 2. Amostras oleosas e 
Ao meu Deus por tudo.

Ao meu pai, Pedro (In memorian) e à minha mãe, Odeth, pela incansável dedicação com minha formação e desenvolvimento.

Ao meu esposo, Marcos, e às minhas filhas, Victória e Anna Clara, pelo amor, apoio, estímulo, confiança e compreensão à minha ausência. 


\section{Agradecimentos}

Ao meu orientador, Professor Ricardo Queiroz Aucélio, pelo estímulo e parceria para a realização deste trabalho.

À minha co-orientadora e amiga, Professora Carmem Lúcia Porto da Silveira, pelo estimulo, confiança e carinho dispensados sempre a mim.

Ao CNPq e à PUC - Rio, pelos auxílios concedidos, sem os quais este trabalho não poderia ter sido realizado.

Ao Professor Norbert Miekeley pelo apoio constante e pelas importantes contribuições durante a realização deste trabalho.

Aos técnicos Maurício Dupim e Álvaro, pelo auxílio competente e dedicado nas análises e no treinamento, pela amizade e tranqüilidade sempre presentes.

Aos alunos, Bárbara e André que, durante sua iniciação científica, contribuíram de forma significativa para a agilidade deste trabalho.

Aos meus pais, meu esposo, minhas filhas, por serem o motivo de todo meu estímulo.

Às minhas amigas Suzana Prado e Mônica de Jesus, pela amizade e encorajamento em todos os momentos.

Aos meus colegas da PUC - Rio, Mônica, Maurício, Ilfran, Bárbara, Daniele, Rodrigo, Álvaro, Heloísa, Paula, Vanessa, André, Anilton e Mariela pela amizade e incentivo.

Aos professores e funcionários do Departamento de Química da PUC - Rio, pela contribuição na minha formação acadêmica.

Aos professores que participaram da Comissão examinadora. 


\section{Resumo}

Souza, Roseli Martins; Aucélio, Ricardo Queiroz. Desenvolvimento de métodos analíticos para determinação de elementos-traço em amostras oleosas e pastosas por ICP OES e ICP-MS. Rio de Janeiro, 2007, 187 p. Tese de Doutorado - Departamento de Química, Pontifícia Universidade Católica do Rio de Janeiro.

Métodos de análise para determinação de elementos-traço em amostras oleosas e pastosas são necessários, por exemplo, no caso do petróleo e em suas frações mais pesadas, para os quais os elementos-traço indicam características do tipo do petróleo, correlacionando-o com a localização geográfica da jazida. Além disso, a quantificação destes elementos tem utilidade nas interpretações geoquímicas relacionadas com maturidade térmica, correlação óleo-óleo e migração primária e secundária do óleo e na obtenção de informações que permitem a criação de estratégias para realizar o refino do óleo, prevenindo contaminação dos catalisadores, corrosão de equipamento e potencial dano ambiental. No caso de óleos e gorduras vegetais, a presença destes elementos, mesmo em baixas concentrações $\left(\mu \mathrm{g} \mathrm{g}^{-1}\right)$, pode acelerar processos oxidativos que geram peróxidos, aldeídos, cetonas, ácidos e epóxidos, que além de causarem a rancidez prematura dos óleos produzem efeitos patológicos no sistema digestivo e potencializam a ação de alguns carcinogênicos. As técnicas espectrométricas baseadas no uso do plasma (ICP OES e ICP-MS) estão entre as mais utilizadas na determinação de elementos-traço nesses tipos de amostra. Porém, os procedimentos de preparação de amostra mais tradicionalmente usados são os que destroem a matriz das amostras, solubilizando-as em ácidos inorgânicos fortes. Neste trabalho, novas estratégias de preparação de amostras de petróleo, óleo diesel, asfaltenos, óleos comestíveis, manteiga e margarina foram desenvolvidas visando à utilização do tradicional procedimento de nebulização de solução para introdução de amostra no plasma. No caso do óleo diesel, a estratégia avaliada foi a de preparação de amostras na forma de emulsão com n-propanol. Nesse caso, um planejamento fatorial indicou que fatores importantes para a determinação de elementos refratários foram tanto a massa de amostra na composição da emulsão (até $25 \%$ em massa) quanto a acidificação da emulsão. As amostras de óleo cru, óleos vegetais e gorduras vegetais foram preparadas como microemulsão em n-propanol. Alternativamente, um 
procedimento de extração assistida por ultra-som foi desenvolvido. A emulsão de óleo cru com n-propanol mostrou ser um bom meio de estabilização dos analitos em solução. No caso da extração ácida assistida por ultra-som para óleo cru, um planejamento fatorial mostrou que os fatores importantes para a determinação de elementos-traço foram, o tempo de aquecimento da amostra, a concentração do ácido nítrico e a exposição ao ultra-som. Para as amostras de óleos e gorduras vegetais, a otimização das proporções de componentes na emulsão com n-propanol proporcionou boa estabilidade e homogeneidade das amostras de óleo de oliva e óleo de soja (6 \% em massa de óleo) e para margarina e manteiga ( $5 \% \mathrm{~m} / \mathrm{m}$ de óleo). Para o método da extração ácida assistida por ultra-som, as condições otimizadas para a preparação da amostra permitiram também a análise por ICP-MS, cujos resultados são discutidos e comparados com os resultados obtidos por ICP OES. Para as amostras de asfaltenos, o procedimento proposto foi o de extração em $\mathrm{HNO}_{3}$ concentrado assistida por ultrasom. Nesse caso, os mesmos parâmetros otimizados para o óleo cru foram usados. Os parâmetros operacionais foram otimizados para obtenção dos parâmetros de mérito, sendo a aplicação dos métodos propostos confirmada por testes de recuperação além de comparação com resultados obtidos com procedimentos tradicionais para esse tipo de amostra. Assim, nessa tese, foram desenvolvidos métodos para a determinação de Cr, Mo, V e Ti em óleo diesel e em óleo combustível, Cd, Co, Cr, Fe, Mn, Mo, Ni, Ti, V e Zn em óleo cru, Fe, Ni e V em asfaltenos. $\mathrm{Cd}, \mathrm{Co}, \mathrm{Cr}, \mathrm{Cu}, \mathrm{Mn}$ e Ni foram determinados em óleos e gorduras comestíveis utilizando preparação de amostra como microemulsão em n-propanol. Já $\mathrm{Al}, \mathrm{Ca}, \mathrm{Cd}, \mathrm{Cu}, \mathrm{Fe}, \mathrm{Mg}, \mathrm{Mn}, \mathrm{Ni}$, V e Zn foram determinados após extração ácida assistida por ultra-som. As análises foram realizadas por calibração externa com padronização interna com Sc (no caso de ICP OES). O método da extração ácida assistida por ultra-som foi utilizado para análise de óleos e gorduras comestíveis por PN-ICP-MS. Nesse caso, utilizaram-se os mesmo parâmetros otimizados para a análise por ICP OES, porém usando o Rh como padr interno. Foram determinados $\mathrm{Cd}, \mathrm{Cu}, \mathrm{Fe}, \mathrm{Mn}, \mathrm{Ni}, \mathrm{Pb}, \mathrm{V}$ e $\mathrm{Zn}$ em óleo de oliva, óleo de soja e margarina. Os limites de detecção dos métodos propostos foram da ordem de $\mu \mathrm{g} \mathrm{g}^{-1}$ e $\mathrm{ng} \mathrm{g}^{-1}$ com recuperações entre 90 e $115 \%$.

\section{Palavras-chave}

Amostras oleosas e pastosas; elementos-traço; ICP OES; ICP-MS; emulsão; microemulsão; extração em meio ácido. 


\section{Abstract}

Souza, Roseli Martins; Aucélio, Ricardo Queiroz. Development of analytical methods for trace elements determinations in oily and viscous samples by ICP OES e ICP-MS. Rio de Janeiro, 2007, 187 p. Tese de Doutorado Departamento de Química, Pontifícia Universidade Católica do Rio de Janeiro.

Analytical methods for the determination of trace elements in oily and viscous samples are necessary, for example, in the case of crude oil and its heavy fractions, where trace elements indicate specific oil characteristics and the geographic location of the natural deposit. Moreover, the quantification of these elements is relevant for geochemical interpretations concerning thermal maturity, oil-oil correlations and primary and secondary oil migration. Knowledge on trace element characteristics of crude oil is also important in oil refining strategies to prevent contamination of catalysts as well as equipment corrosion, and to minimize environmental pollution. In the case of vegetal oils and fats, the presence of certain elements, even in low concentrations $\left(\mu \mathrm{g} \mathrm{g}^{-1}\right)$; can speed up oxidative processes and premature rancidity of oils, which may cause pathological effects in the digestive system, including cancer. Spectrometric techniques based on inductively coupled plasma (ICP OES and ICPMS) are extensively used for determination of trace elements in such type of samples due to their sensitivities and multielemental features. In the case of oils and fats, the samples have to be generally decomposed by wet-ashing procedures with mineral acids, prior to the elemental determinations. In this work, new strategies for sample preparation of crude oil, diesel, asphaltenes, edible oils, butter and margarine have been developed, which allow the use of the traditional procedure for sample introduction into the ICP by solution nebulization. In the case of diesel oil, the proposed strategy was the preparation of samples as emulsions with n-propanol. Factorial design studies showed that the important factors for the quantitative determination of refractory metals were the sample amount in the emulsion (up to $25 \%$ in weight) and the acidification of the emulsion. Crude oil samples, vegetable oils and fats have been prepared as n-propanol microemulsions. Alternatively, an ultrasound-assisted trace metal extraction procedure was developed. The crude oil emulsions with n-propanol were found to be a good environment to keep the analytes in solution. In the case of the ultrasound-assisted acid extraction for crude oil, a 
factorial design indicated that the important factors for the determination of trace elements have been the sample heating time, the nitric acid concentration and the ultrasound exposure time. For vegetable oils and fats, the optimization of the npropanol emulsion components provided good stability and homogeneity olive and soy oils ( $6 \%$ of oil in weight) and for margarine and butter ( $5 \%$ of oil in weight). For the ultrasound-assisted acid extraction, the optimized conditions for sample preparation also allowed the analysis of the extracts by ICP-MS, which results were compared with the ones obtained by ICP OES. For asphaltene samples, the proposed procedure was the ultrasound- assisted acid extraction in concentrated $\mathrm{HNO}_{3}$ using similar conditions employed for crude oil. The figures of merit for the proposed methods were achieved under optimized experimental and instrumental conditions and validations were performed by analysis of certified reference materials (when available), recovery tests, and by comparison of results obtained using traditional sample preparation procedures for this type of sample. Methods for the determination of $\mathrm{Cr}$, Mo, $\mathrm{V}$ and $\mathrm{Ti}$ in fuel and diesel oils, for $\mathrm{Cd}, \mathrm{Co}, \mathrm{Cr}, \mathrm{Fe}, \mathrm{Mn}, \mathrm{Mg}, \mathrm{Ni}, \mathrm{Ti}, \mathrm{V}$ and $\mathrm{Zn}$ in crude oil and for $\mathrm{Fe}, \mathrm{Ni}$ and $\mathrm{V}$ in asphaltenes have been developed. $\mathrm{Cd}, \mathrm{Co}, \mathrm{Cr}$, $\mathrm{Cu}, \mathrm{Mn}$ and $\mathrm{Ni}$ in edible oils and fats have been determined using the microemulsification in n-propanol, while $\mathrm{Al}, \mathrm{Ca}, \mathrm{Cd}, \mathrm{Cu}, \mathrm{Fe}, \mathrm{Mg}, \mathrm{Mn}, \mathrm{Ni}, \mathrm{V}$ and $\mathrm{Zn}$ have been determined after acid extraction in $\mathrm{HNO}_{3}$. The analyses were carried out by using the external calibration method with internal standardization by Sc (in the case of ICP OES). The ultrasound-assisted acid extraction method for the analysis of edible oils and fats by ICP OES was also tested for pneumatic nebulization in ICPMS, allowing the determination of $\mathrm{Cd}, \mathrm{Cu}, \mathrm{Fe}, \mathrm{Mn}, \mathrm{Ni}, \mathrm{Pb}, \mathrm{V}$ and $\mathrm{Zn}$ in olive oil, soy oil and margarine. The limits of detection of the proposed methods are of the order of $\mu \mathrm{g} \mathrm{g}^{-1}$ and $\mathrm{ng} \mathrm{g}^{-1}$, with recoveries between 90 and $115 \%$.

\section{Key-words}

Oily and viscous samples; trace elements; ICP OES; ICP-MS; emulsion; microemulsion; acid extraction. 


\section{Sumário}

1 Introdução 21

1.1. Petróleo: Análise e especificações 23

1.1.1 Constituintes não-destiláveis 26

1.1.2 Classificação do petróleo 27

1.1.3 Elementos-traço em petróleo e sua importância 29

1.2 Óleos e gorduras comestíveis $\quad 36$

1.2.1 Fatores biológicos importantes dos metais 37

2 Análise de amostras de óleo e de gorduras por espectrometria atômica 41

2.1. Desempenho das metodologias analíticas espectrométricas para a determinação de metais-traço em amostras de petróleo e derivados, e óleos e gorduras comestíveis.

2.1.1 Espectrometria de Absorção Atômica (AAS) 42

2.1.2 Espectrometrias de Fluorescência Atômica Óptica e de Raios-X 43

2.1.3 Espectrometria de Emissão Óptica em ICP 44

2.1.4 Espectrometria de Massa em ICP 45

2.2. Técnicas de preparação de amostras de petróleo e derivados e óleos e gorduras comestíveis 46

2.2.1 Calcinação 47

2.2.2 Diluição direta em solventes orgânicos 49

2.2.3 Dissolução ácida da amostra 52

2.2.4 Extração ácida $\quad 55$

2.2.5 Extração ácida assistida por ultra-som 57

2.2.6 Emulsificação da amostra 59

2.3 Objetivos 62

3 Materiais e métodos $\quad 64$

3.1 Equipamentos 64 
3.1.1 Espectrometria de Emissão Óptica com Plasma Indutivamente Acoplado

3.1.2 Espectrometria de Massa com Plasma Indutivamente Acoplado 66

3.2 Reagentes, Soluções e Materiais 69

3.3 Procedimentos gerais $\quad 70$

3.3.1 Óleo Diesel $\quad 70$

3.3.2 Óleo Cru 73

3.3.3 Asfaltenos $\quad 76$

3.3.4 Óleos e gorduras comestíveis $\quad 78$

3.3.4.1 Emulsificação da amostra com Triton X-100 78

4 Resultados e discussão: Estudos preliminares 81

4.1 Interferências associadas às técnicas baseadas no uso do ICP 81

4.1.1 Interferências espectrais 81

4.2 Minimização de interferências não-espectrais $\quad 87$

4.2.1 Padronização interna $\quad 87$

4.2.2 Introdução de oxigênio no gás carreador e no gás auxiliar $\quad 88$

4.2.3 Acidificação de amostras 91

4.3 Estudo das condições de cavitação do banho ultra-sônico 92

5 Resultados e discussão: Comparação entre dois procedimentos de emulsificação para a determinação de $\mathrm{Cr}$, Mo, V e Ti em óleo diesel e óleo combustível por ICP OES utilizando planejamento fatorial

5.1 Otimizações experimentais 97

5.1.1 Composição das emulsões 97

5.1.2 Condições experimentais e instrumentais 98

5.2 Planejamento fatorial completo $2^{3} \quad 99$

5.3 Parâmetros de mérito 103

5.4 Desempenho analítico e considerações finais sobre o método 105

6 Determinação de metais-traço em amostras de óleo cru por dois diferentes métodos de preparação: emulsificação sem detergente 
e extração ácida assistida por ultra-som.

6.1 Determinação de $\mathrm{Cd}$, Co, Cr, Fe, Mo, Ni, Ti, V e Zn em óleo cru usando ICP OES e introdução da amostra como microemulsão sem detergente

6.1.1 Otimização das microemulsões sem detergente

6.1.2 Estabilidade dos analitos na microemulsão

6.1.3 Desempenho analítico

6.1.4 Considerações sobre a utilização de microemulsão sem detergente para óleo cru

6.1.5 Estudos envolvendo ICP-MS

6.2 Determinação de elementos-traço em óleo cru por ICP OES usando extração ácida assistida por ultra-som

6.2.1 Variáveis experimentais críticas para extração ácida do óleo assistida por ultra-som

6.2.2 Planejamento fatorial

6.2.3 Avaliação da influência da posição dos tubos no banho ultra-sônico na extração ácida

6.2.4 Parâmetros de mérito

6.2.6 Considerações sobre o uso do procedimento de extração ácida assistida por ultra-som de metais-traço de óleo cru

6.3 Comparativo entre os dois métodos propostos, emulsificação sem detergente e extração ácida, em termos de aplicação na determinação de elementos-traço presentes em óleo cru

7 Determinação de $\mathrm{Fe}, \mathrm{Ni}$ e $\mathrm{V}$ em asfaltenos por extração ácida assistida por ultra-som usando ICP OES

7.2 Condições experimentais e instrumentais

7.3 Parâmetros de mérito

7.3.1 Sensibilidade e precisão

7.4 Considerações sobre o método de extração assistida por 
8 Determinação de elementos-traço em óleos e gorduras comestíveis

8.1 Determinação de $\mathrm{Cd}, \mathrm{Co}, \mathrm{Cr}, \mathrm{Cu}, \mathrm{Mn}$ e Ni em óleos vegetais, margarina e manteiga após emulsificação com n-propanol e água por ICP OES

8.1.1 Otimização das condições experimentais

8.1.2 Estudo de correlação de sinais para as microemulsões

8.1.3 Análise de óleos e gorduras

8.1.4 Considerações finais sobre o método proposto

8.2 Determinação de Al, Ca, Cd, Cu, Cr, Fe, Mg, Mn, Mo, Ni, Pb,

Ti, V e Zn em óleos vegetais e margarina através da extração ácida assistida por ultra-som pelas técnicas de ICP OES e de ICP-MS

8.2.1 Parâmetros de mérito

8.2.2 Aplicação analítica

9 Conclusões 


\section{Lista de Figuras}

Figura 1 - Espectrômetro de Emissão Óptica, P1000 (Perkin Elmer), do laboratório de ICP OES da PUC-Rio.

Figura 2 - Espectrômetro de Massa, Elan 5000 (Perkin Elmer-Sciex), do laboratório de ICP-MS da PUC-Rio.

Figura 3 - Calibração das vazões de oxigênio no rotâmetro

Figura 4. Esquema de montagem para introdução de

oxigênio na vazão de argônio auxiliar

Figura 5 - Banho ultra-sônico utilizado neste trabalho

Figura 6. Resultados do teste de erosão do alumínio pela cavitação do banho de ultra-som em função do tempo.

Figura 7. Microemulsão de óleo cru em n-propanol

Figura 8. Variação da intensidade de emissão do analito (Mo, Zn, Cd, Ti, $\mathrm{Ni}, \mathrm{V}, \mathrm{Fe}, \mathrm{Mn}, \mathrm{Cr}$ ) em função do tempo, em óleo cru dissolvido em tolueno e preparado como microemulsão em n-propanol, fortificados com padrão organometálico $\left(5 \mu \mathrm{g} \mathrm{g}^{-1}\right)$.

Figura 9. Recuperações de elementos traço de oleo cru em função do tempo de sonicação usando ácido nítrico como meio líquido em duas diferentes concentrações (6 ou $16 \mathrm{~mol} \mathrm{~L}^{-1}$ ) : metais refratários $\mathrm{Cd}, \mathrm{Fe}, \mathrm{Mn}$, Ni e Zn. Tempo de pré-aquecimento $(30 \mathrm{~min})$ a $85{ }^{\circ} \mathrm{C}$.

Figura 10. Recuperações de elemento traço de oleo cru em função do tempo de sonicação usando ácido nítrico como meio líquido em duas diferentes concentrações (6 ou $16 \mathrm{~mol} \mathrm{~L}^{-1}$ ): metais refratários $\mathrm{Cr}$, Mo, $\mathrm{V}$ e Ti. Tempo de pré-aquecimento $(30 \mathrm{~min})$ a $85{ }^{\circ} \mathrm{C}$.

Figura 11. Estudo comparativo de desempenho entre Ultra-som (US) e Vórtex na extração de Ni e V do NIST 1634c.

Figura 12. Emulsões em Triton X-100 de óleo de oliva e de óleo de soja; e microemulsões em n-propanol de óleo de oliva e de óleo de soja

Figura 13. Emulsões de margarina em Triton X-100, bem como em n-propanol 
Figura 14. Estudo comparativo de sinais obtidos para $\mathrm{Cd}, \mathrm{Co}, \mathrm{Cr}, \mathrm{Cu}, \mathrm{Mn}$ e Ni (10 $\left.\mu \mathrm{g} \mathrm{g}^{-1}\right)$ em óleo de oliva (azeite) e óleo de soja emulsificados em

n-propanol, em solução aquosa e em solução aquosa de n-propanol. 


\section{Lista de Tabelas}

Tabela 1: Análise elementar de óleo cru típico (fração de massa em \%) ${ }^{8} \quad 26$

Tabela 2 - Parâmetros operacionais do ICP-MS $\quad 68$

Tabela 3 - Linhas selecionadas para os elementos a serem determinados por $\quad 83$ ICP OES - Al, Cd, Co, Cr, Cu, Fe, Mg, Mn, Mo, Ni, Ti, V e Zn

Tabela 4. Razão m/z dos elementos determinados por ICP-MS. 85

Tabela 5. Variáveis e seus níveis para o planejamento fatorial $2^{3} \quad 96$

Tabela 6. Planejamento experimental e resultados do planejamento fatorial $2^{3}$ para emulsão com detergente preparada com Triton X-100.

100

Tabela 7. Planejamento experimental e resultados do planejamento fatorial $2^{3}$ para emulsão sem detergente preparada com n-Propanol

Tabela 8. Efeitos principal e de interação e seus erros-padrão (\%) para

emulsão em Triton X-100

102

Tabela 9. Efeitos principal e de interação e seus erros-padrão (\%) para emulsão em n-propanol

Tabela 10. Parâmetros analíticos de mérito

Tabela 11. Recuperações percentuais para alguns elementos usando diferentes procedimentos de preparação da amostra $(n=4 ; p=95 \%$, nível de fortificação: $5 \mu \mathrm{g} \mathrm{g}^{-1}$ concentração final do analito).

Tabela 12. Limites de detecção e quantificação para os analitos usando o método de microemulsão em n-propanol por ICP OES na microemulsão

Tabela 13. Recuperações $(n=3)$ dos analitos para MRC NIST 1634c

Tabela 14. Recuperações dos analitos $(n=3, t=95 \%)$ para óleos crus fortificados com Conostan S-21 (5 $\left.\mu \mathrm{g} \mathrm{g}^{-1}\right)$

Tabela 15. Planejamento experimental e resultados do planejamento fatorial $2^{3}$ para extração ácida assistida por ultra-som

Tabela 16. Efeitos principaisl e de interação e seus desvios-padrão (\%) para metais-traço por extração ácida assistida por ultra-som

Tabela 17. Limites de detecção e quantificação para os analitos usando o método de extração ácida por ICP OES na solução ácida

Tabela 18. Recuperações $(n=3)$ em MRC NIST 1634c e em amostras de 
óleo cru fortificadas $\left(5 \mu \mathrm{g} \mathrm{g}^{-1}\right)$ comparando a metodologia da extração ácida com a decomposição ácida

Tabela 19 - Limites de detecção em $\mu \mathrm{g} \mathrm{g}^{-1}$, obtidos para Cd, Co, Cr, Fe, Mn, Mo, Ni, Ti, V e Zn na amostra pelas metodologias desenvolvidas (emulsão em n-propanol e extração ácida assistida por ultra-som) e pela já estabelecida (decomposição ácida por micro-ondas)

Tabela 20 - Determinação da concentração de Cd, Fe, Mn, Mo em amostra original de óleo cru I ( $\mathrm{n}=3)$

Tabela 21- Determinação da concentração de Ni, Ti,V e Zn em amostra original de óleo cru I ( $\mathrm{n}=3)$

Tabela 22. Condições experimentais utilizadas para extração assistida por ultra-som de $\mathrm{Fe}$, Ni e $\mathrm{V}$ em asfalteno.

Tabela 23- Estudo comparativo de parâmetros analíticos de mérito obtidos para amostra de asfaltenos entre o método de extração ácida e da diluição direta Tabela 24. Concentrações $\left(\mu \mathrm{g} \mathrm{g}^{-1}\right), \mathrm{n}=3$ para $\mathrm{Fe}, \mathrm{Ni}$ e V determinados em asfaltenos

Tabela 25 - Comparação de resultados de recuperação $(n=3)$ de metais-traço de MRC NIST 1634c obtidos com extração ácida assistida por ultra-som (20 min) e por agitação vórtex (50 min).

Tabela 26 - Determinação das concentrações $\left(\mu \mathrm{g} \mathrm{g}^{-1}\right)$ de $\mathrm{Cd}, \mathrm{Co}, \mathrm{Cr}, \mathrm{Cu}, \mathrm{Mn}$ e Ni nas amostras de óleo de oliva, óleo de soja, margarina e manteiga

Tabela 27. Parâmetros analíticos de mérito para $\mathrm{Cd}, \mathrm{Co}, \mathrm{Cr}, \mathrm{Cu}, \mathrm{Mn}$ e Ni nas microemulsões, emulsões e nas amostras (fator de diluição de 20 X)

Tabela 28. Equações de regressão linear e coeficientes de determinação das curvas de calibração preparadas com padrões inorgânicos

Tabela 29 - Recuperações do analito (\%) para óleos comestíveis e gorduras fortificados $(95 \%, \mathrm{n}=4)$

Tabela 30 - Condições experimentais utilizadas para extração assistida por ultra-som de elementos-traço em óleos e gordura comestíveis (5,00 g de óleo ou 0,50 g de margarina para $10 \mathrm{~mL}$ de solução ácido nítrico).

Tabela 31. Parâmetros de mérito das curvas de calibração externa com padrões inorgânicos em soluções de $\mathrm{HNO}_{3} 2,7 \mathrm{~mol} \mathrm{~L}{ }^{-1}$ e por soluções de Triton X-100 por ICP OES 
Tabela 32 - Parâmetros de mérito das curvas de calibração externa com padrões inorgânicos em soluções de $\mathrm{HNO}_{3} 2,7$ mol L-1 por ICP-MS.

Tabela 33- Estudo comparativo de parâmetros analíticos de mérito obtidas para o método de extração ácida em óleo de oliva, óleo de soja e margarina por ICP OES e por ICP-MS

Tabela 34 - Concentrações $\left(\mu \mathrm{g} \mathrm{g}^{-1}\right)$ de elementos-traço nas amostras de óleo de oliva, óleo de soja e margarina por ICP OES e ICP-MS

Tabela 35- Estudo comparativo de parâmetros analíticos de mérito obtidos para o método de extração ácida em óleo de oliva, óleo de soja e margarina entre ICP OES e ICP-MS

Tabela 36. Resumo das metodologias desenvolvidas 


\section{Lista de Abreviatura}

ICP OES - Espectrometria de Emissão Óptica com Plasma Indutivamente Acoplado.

ICP-MS - Espectrometria de Massa com Plasma Indutivamente Acoplado.

ICP - Plasma Indutivamente acoplado.

$\mathrm{RF}$ - Radiofreqüência

DPEP - Deoxofiloeritoetioporfirina

SOD - Superóxido Dismutase

AAS - Espectrometria de Absorção Atômica

FAAS - Espectrometria de Absorção Atômica com Chama

ET - Atomizador Eletrotérmico

ET AAS - Espectrometria de Absorção Atômica com Atomizador Eletrotérmico

HR-CS ET AAS - Espectrometria de Absorção Atômica de Alta Resolução com Atomização Eletrotérmica e Fonte Contínua

SOAP - Programa para Análise de Óleos por Espectrometria

AFRX - Espectrometria de Fluorescência Atômica de Raios X

AOCS - American Oils Chemists Society

CV AAS - Espectrometria de Absorção Atômica com Vapor Frio

LD - Limite de Detecção

MIBK - Metil Iso Butil Cetona

US - Ultra-som

USN - Nebulizador Ultra-Sônico

LQ - Limite de Quantificação

ETV-ICP-MS - Espectrometria de Massa com Plasma Indutivamente Acoplado com Vaporização Eletrotérmica

DC - Corrente Direta

SDS - Dodecil Sulfato de Sódio

QAV - Querosene de Aviação

MRC - Material de Referência Certificado

BEC - Concentração Equivalente ao Branco

PCA - Análise de Componentes Principais 
FA - Análise de Fatores

RSD - Desvio Padrão Relativo

BLH - Balanço Lipofílico-Hidrofílico

PTFE - Politetrafluoroetileno

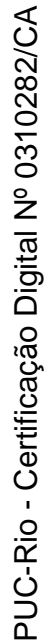

